\title{
mant \\ A High-Performance Deep Neural Network Model for BI-RADS Classification of Screening Mammography
}

\author{
Kuen-Jang Tsai ${ }^{1,2}{ }^{,}$Mei-Chun Chou ${ }^{3}$, Hao-Ming Li ${ }^{3}{ }^{\oplus}$, Shin-Tso Liu ${ }^{3}$, Jung-Hsiu Hsu ${ }^{3}$, Wei-Cheng Yeh ${ }^{4}$, \\ Chao-Ming Hung ${ }^{1}$, Cheng-Yu Yeh ${ }^{5, *}$ and Shaw-Hwa Hwang ${ }^{6}$
}

1 Department of General Surgey, E-Da Cancer Hospital, Yanchao Dist., Kaohsiung 82445, Taiwan; tsai560612@gmail.com (K.-J.T.); ed100647@edah.org.tw (C.-M.H.)

2 College of Medicine, I-Shou University, Yanchao Dist., Kaohsiung 82445, Taiwan

3 Department of Radiology, E-Da Hospital, Yanchao Dist., Kaohsiung 82445, Taiwan; meichun@gmail.com (M.-C.C.); 1hm0323@gmail.com (H.-M.L.); ed106088@edah.org.tw (S.-T.L.); wenbear0330@gmail.com (J.-H.H.)

4 Department of Radiology, E-Da Cancer Hospital, Yanchao Dist., Kaohsiung 82445, Taiwan; ywcywcywcywc@gmail.com

5 Department of Electrical Engineering, National Chin-Yi University of Technology, Taichung 41170, Taiwan

6 Department of Electrical and Computer Engineering, National Yang Ming Chiao Tung University, Hsinchu 30010, Taiwan; hsf@nctu.edu.tw

* Correspondence: cy.yeh@ncut.edu.tw

Citation: Tsai, K.-J.; Chou, M.-C.; Li, H.-M.; Liu, S.-T.; Hsu, J.-H.; Yeh, W.-C.; Hung, C.-M.; Yeh, C.-Y.; Hwang, S.-H. A High-Performance Deep Neural Network Model for BI-RADS Classification of Screening Mammography. Sensors 2022, 22, 1160. https://doi.org/10.3390/ s22031160

Academic Editors: Jian Cao, Chintan Bhatt, Monowar H. Bhuyan, Behnaz Ghoraani and Mukesh Prasad

Received: 5 January 2022

Accepted: 31 January 2022

Published: 3 February 2022

Publisher's Note: MDPI stays neutral with regard to jurisdictional claims in published maps and institutional affiliations.

Copyright: (C) 2022 by the authors. Licensee MDPI, Basel, Switzerland. This article is an open access article distributed under the terms and conditions of the Creative Commons Attribution (CC BY) license (https:// creativecommons.org/licenses/by/ $4.0 /)$.

\begin{abstract}
Globally, the incidence rate for breast cancer ranks first. Treatment for early-stage breast cancer is highly cost effective. Five-year survival rate for stage 0-2 breast cancer exceeds $90 \%$. Screening mammography has been acknowledged as the most reliable way to diagnose breast cancer at an early stage. Taiwan government has been urging women without any symptoms, aged between 45 and 69 , to have a screening mammogram bi-yearly. This brings about a large workload for radiologists. In light of this, this paper presents a deep neural network (DNN)-based model as an efficient and reliable tool to assist radiologists with mammographic interpretation. For the first time in the literature, mammograms are completely classified into BI-RADS categories $0,1,2,3$, $4 \mathrm{~A}, 4 \mathrm{~B}, 4 \mathrm{C}$ and 5 . The proposed model was trained using block-based images segmented from a mammogram dataset of our own. A block-based image was applied to the model as an input, and a BI-RADS category was predicted as an output. At the end of this paper, the outperformance of this work is demonstrated by an overall accuracy of $94.22 \%$, an average sensitivity of $95.31 \%$, an average specificity of $99.15 \%$ and an area under curve (AUC) of 0.9723 . When applied to breast cancer screening for Asian women who are more likely to have dense breasts, this model is expected to give a higher accuracy than others in the literature, since it was trained using mammograms taken from Taiwanese women.
\end{abstract}

Keywords: screening mammography; breast imaging reporting and data system (BI-RADS); image classification; deep neural network (DNN); deep learning

\section{Introduction}

Globally, the incidence rate for breast cancer ranks first [1]. A recent report [2] indicates that more than 10,000 Taiwanese women are diagnosed as having breast cancer, and more than 2000 died of breast cancer in 2018. As a matter of fact, treatments for early-stage breast cancer are effective. The 5-year survival rate for stage 0-2 breast cancer exceeds $90 \%$, while it falls below $25 \%$ for stage 4 [3]. Screening mammography has been acknowledged as the most reliable way to detect breast cancer at an early stage, particularly in detecting grouped microcalcification lesions. For years, the Taiwanese government has been urging women without any symptoms, aged between 45 and 69, to have a screening mammogram on a biennial basis. A great number of mammograms are collected in a large-scale mammography 
screening program and need to be interpreted by well-qualified but overloaded radiologists. Hence, there is definitely an unmet need to develop AI models to assist radiologists with mammographic interpretation, and AI model development requires interdisciplinary research that integrates medical science and engineering.

Routine screening mammography consists of the cranio-caudal (CC) view and the mediolateral-oblique (MLO) view of each breast of a woman, that is, the LCC, RCC, LMLO and RMLO views in total. Developed by the American College of Radiology (ACR), the Breast Imaging Reporting and Data System (BI-RADS) [4] lexicon is used to standardize the reporting of mammographic findings, assessment categories and follow-up management, and communication between radiologists and referring physicians can be facilitated accordingly.

As referenced previously, it takes radiologists an enormous amount of effort to interpret screening mammograms. Therefore, a great amount of computer-aided detection (CAD) systems have been developed to improve the efficiency of mammogram interpretation. Recently, deep learning models have been used to measure the likelihood of cancer from a mammogram [5-22], some of which were designed to detect and classify microcalcifications or calcified lesions [8-10], mass lesions [11-14] and even all the contained lesions [15-22]. Breast lesions found in mammograms are mainly classified into normal, benign and malignant [15-20] and are further classified as normal, benign calcification, benign mass, malignant calcification and malignant mass [21,22].

BI-RADS classification is frequently used in breast cancer screening. Therefore, there is definitely a necessity to develop AI models for efficient and reliable BI-RADS classification. However, little has been reported on this issue in the literature so far, mainly due to an inadequate number of open-access mammogram datasets. For example, breast masses were classified incompletely into BI-RADS categories $2-5$ using a computer-aided diagnosis system [13] where merely 300 mammograms were employed as training data, and another 200 mammograms were employed as testing data.

Accordingly, this paper presents a deep learning model to address the BI-RADSclassification issue. Breast lesions were classified into categories $0,1,2,3,4 \mathrm{~A}, 4 \mathrm{~B}, 4 \mathrm{C}$ and 5 , but excluding category 6 , which indicates a known biopsy-proven malignancy. For the first time in the literature, breast lesions can be completely classified using a deep learning model that was well trained by a mammogram dataset of our own. For the purpose of model training, all the lesions contained were labeled and classified by six well-qualified radiologists, as will be detailed below.

It is worth mentioning that this work can provide at least three benefits for medical industries. First, this developed tool can assist radiologists with mammographic interpretation in clinical works and can improve the efficiency of mammogram interpretation as well. Second, the workload of radiologists can be significantly eased, particularly when interpreting mammograms in a large-scale breast cancer screening program. Third, the tool can assist general physicians to interpret mammograms due to there being a shortage of radiologists or breast surgeons in most remote areas.

This paper is outlined as follows. Section 2 describes a labeled and annotated mammogram dataset for training purposes. Section 3 presents a deep neural network (DNN)-based model for BI-RADS classification. Experimental results and discussions are given in Section 4. Finally, Section 5 concludes this study.

\section{Materials and Lesion Annotation}

Firstly, Table 1 gives the complete BI-RADS categories, the respective description and assessment of mammography [23]. As can be found therein, category 4 is further subcategorized into categories $4 \mathrm{~A}, 4 \mathrm{~B}$ and $4 \mathrm{C}$ to indicate the different levels of malignancy suspicion.

The digital mammogram dataset employed in this work is provided by the E-Da hospital, Taiwan. The dataset is composed of up to 5733 mammograms of 1490 patients, including 1434 LCC, 1436 RCC, 1433 LMLO and 1430 RMLO views, within the time frame 
of 2004 and 2010. This study was approved by a local institutional review board (EMRP108-142), and informed consent was waived. This is simply because there is no personal identifiable data in the dataset, since all the personal data were deleted.

Table 1. Description and assessment of BI-RADS categories for mammograms.

\begin{tabular}{|c|c|c|c|}
\hline BI-RADS & Definition & Management & $\begin{array}{l}\text { Likelihood of } \\
\text { Cancer }\end{array}$ \\
\hline 0 & $\begin{array}{l}\text { Incomplete, need additional } \\
\text { imaging evaluation }\end{array}$ & $\begin{array}{l}\text { Recall for additional } \\
\text { imaging and/or awaiting } \\
\text { prior examinations }\end{array}$ & - \\
\hline 1 & Negative (normal) & Routine screening & $0 \%$ \\
\hline 2 & Benign & Routine screening & $0 \%$ \\
\hline 3 & Probably benign & $\begin{array}{l}\text { Short-interval follow-up or } \\
\text { continued }\end{array}$ & $>0 \%$ to $\leq 2 \%$ \\
\hline $4 \mathrm{~A}$ & Low suspicion of malignancy & Tissue diagnosis & $>2 \%$ to $\leq 10 \%$ \\
\hline $4 \mathrm{~B}$ & $\begin{array}{l}\text { Moderate suspicion of } \\
\text { malignancy }\end{array}$ & Tissue diagnosis & $>10 \%$ to $\leq 50 \%$ \\
\hline $4 C$ & $\begin{array}{l}\text { High suspicion of } \\
\text { malignancy }\end{array}$ & Tissue diagnosis & $>50 \%$ to $<95 \%$ \\
\hline 5 & $\begin{array}{l}\text { Highly suggestive of } \\
\text { malignancy }\end{array}$ & Tissue diagnosis & $\geq 95 \%$ \\
\hline 6 & $\begin{array}{l}\text { Known biopsy-proven } \\
\text { malignancy }\end{array}$ & $\begin{array}{l}\text { Surgical excision when } \\
\text { clinically appropriate }\end{array}$ & $100 \%$ \\
\hline
\end{tabular}

To facilitate data preprocessing, an easy-to-use tool was exclusively developed for users to label the lesion in each mammogram. Once the image labeling was completed, an interface, as illustrated in Figure 1, appeared to give users detailed annotation. In this work, all the lesions in the mammograms were labeled by a total of six qualified radiologists of the E-Da hospital. The annotation was saved as a JSON file. For illustrative purposes, Figure 2 gives a BI-RADS category $4 \mathrm{C}$ mammogram with a labeled lesion and shows a JSON file that saved the annotation in Figure 1.

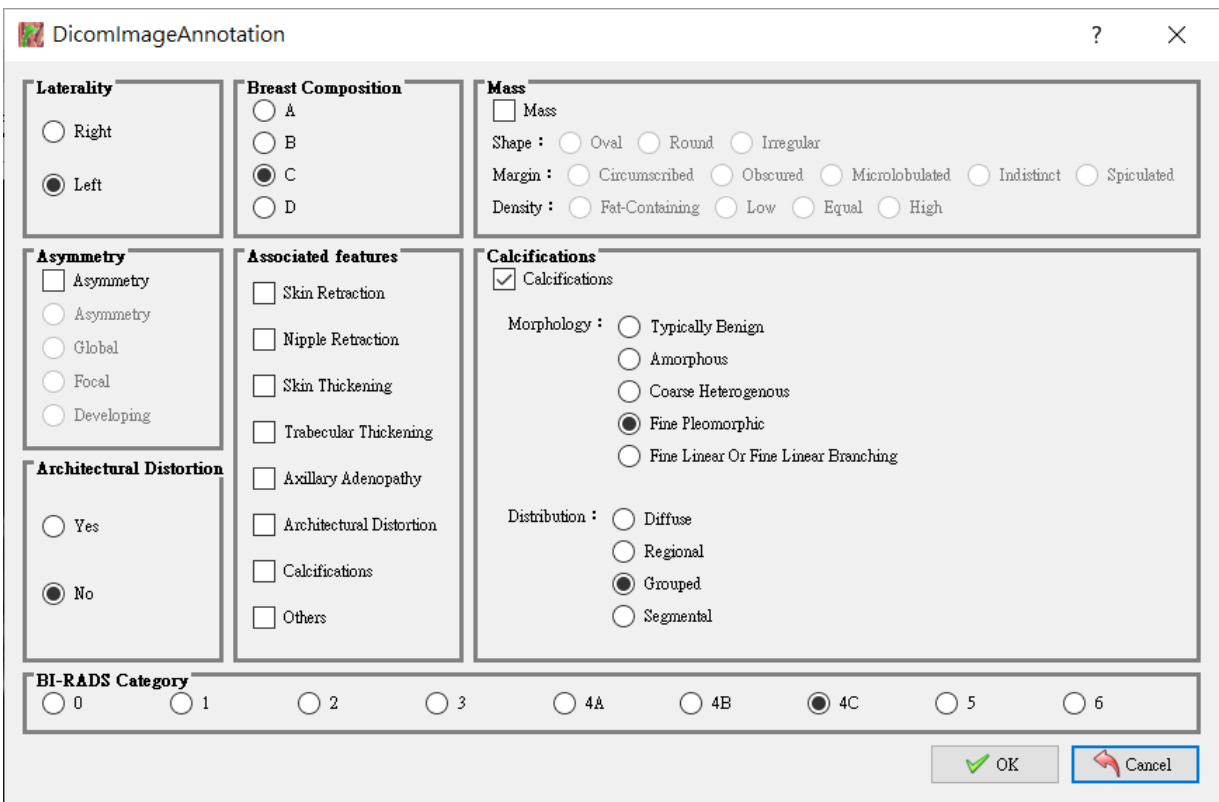

Figure 1. An interface for breast lesion annotation. 


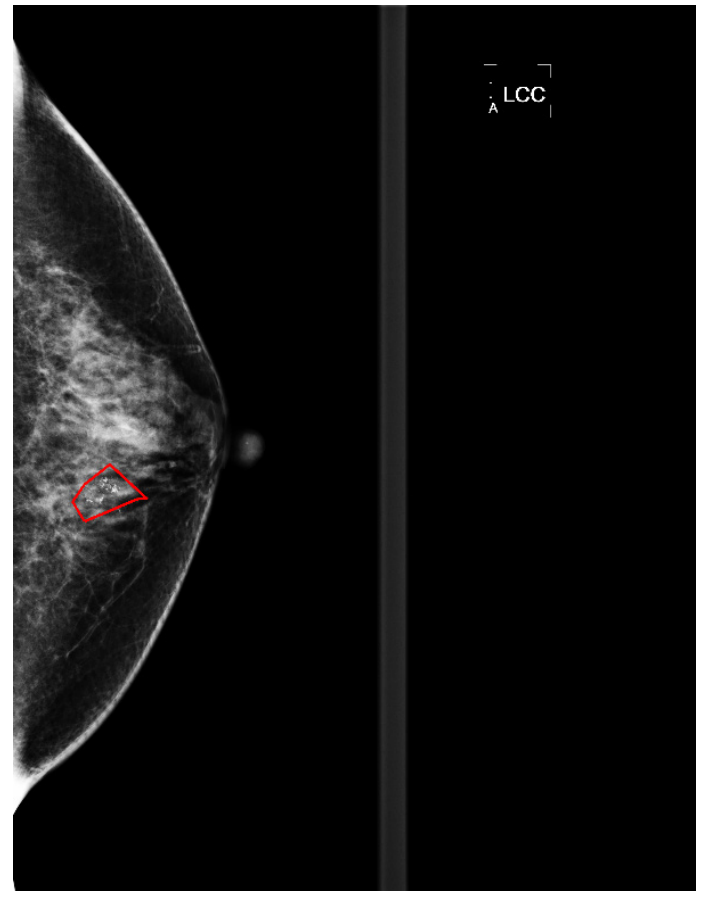

(a)

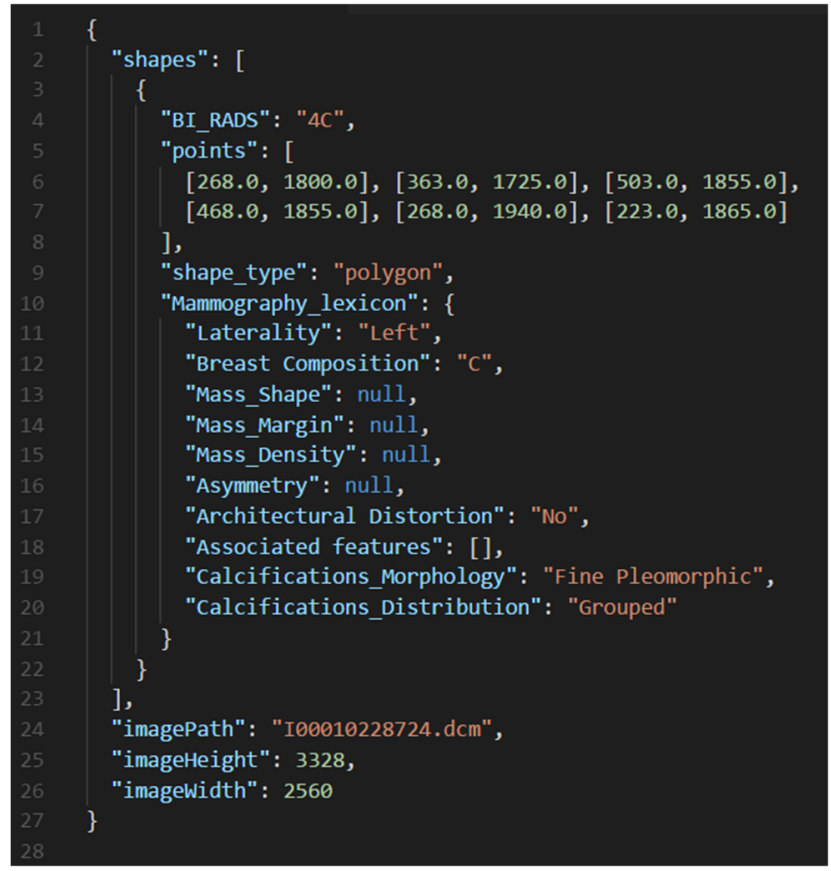

(b)

Figure 2. (a) A BI-RADS category $4 \mathrm{C}$ mammogram with a labeled lesion and (b) a JSON file that saved the annotation in (a).

Table 2 gives the statistics on the number of lesion annotations. As can be found therein, there is no annotation in BI-RADS category 1 , simply because category 1 means that the breast tissue looked healthy, and there was no need to annotate accordingly. Additionally, there is a maximum of 8 annotations in a mammogram and a total of 4557 annotations for all the mammograms in this work.

Table 2. Number of lesion annotations in each BI-RADS category.

\begin{tabular}{cc}
\hline BI-RADS & Number of Annotations \\
\hline 0 & 520 \\
1 & 0 \\
2 & 2125 \\
3 & 847 \\
$4 \mathrm{~A}$ & 367 \\
$4 \mathrm{~B}$ & 277 \\
$4 \mathrm{C}$ & 217 \\
5 & 204 \\
Overall & 4557 \\
\hline
\end{tabular}

\section{Methodology and Model}

This paper presents a DNN-based model to classify mammograms into categories 0 , $1,2,3,4 \mathrm{~A}, 4 \mathrm{~B}, 4 \mathrm{C}$ and 5 , but excluding category 6 , since category 6 is used to represent a female diagnosed with breast cancer. As illustrated in Figure 3, the model was trained using block-based images segmented from the dataset. A block-based image was applied to the model as an input, and a category was assigned as an output. In this manner, the feature maps of the block-based images were correlated with the BI-RADS categories. 


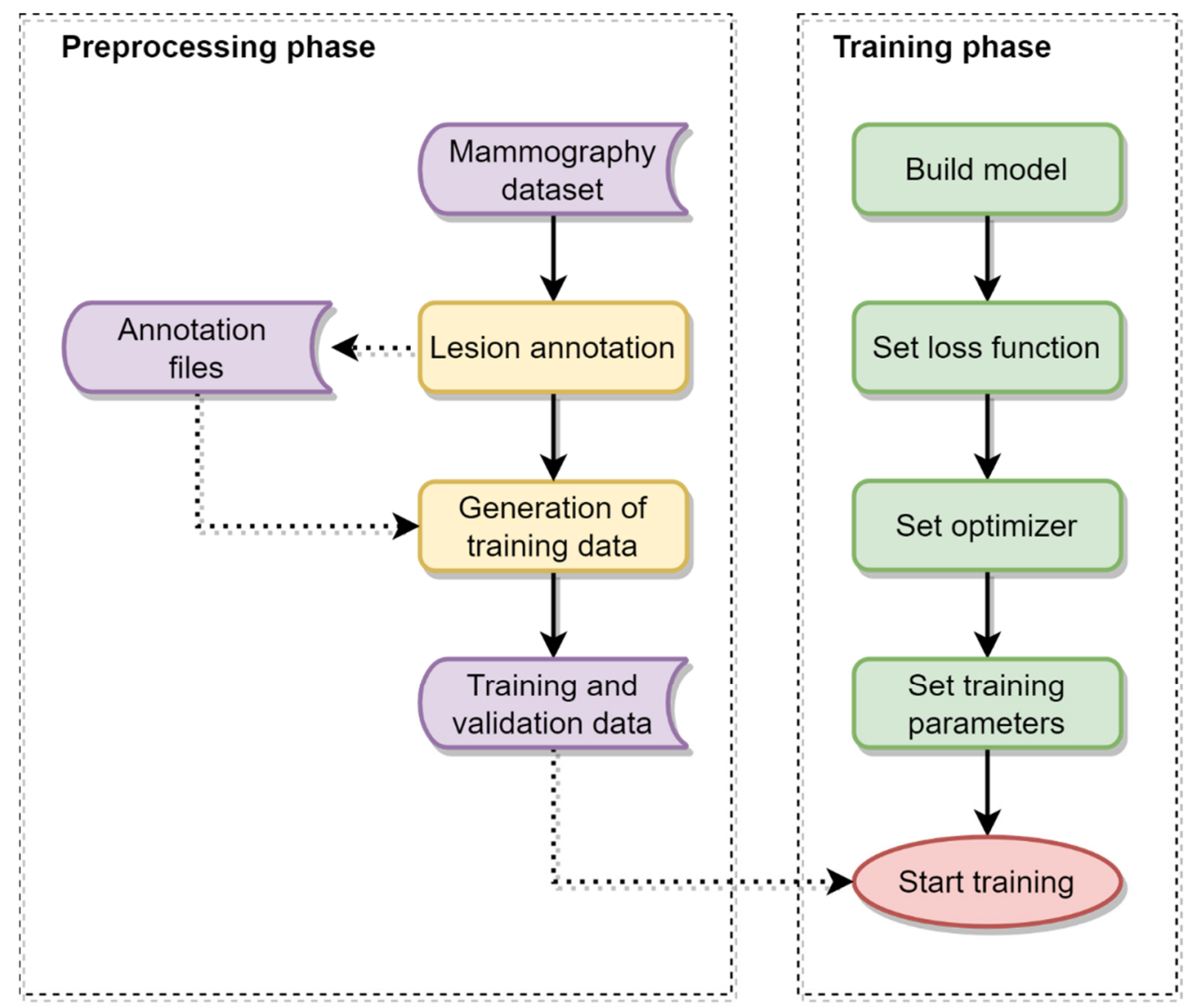

Figure 3. Flowcharts of the preprocessing and training phase in this work.

The DNN-based model has the following advantages. It was well trained using a multitude of block images, and it is the first time that mammograms were classified into eight BI-RADS categories for the sake of completeness in the literature. Finally, breast lesions can be reliably located and efficiently classified to allow the radiologists to speed up mammogram interpretation. The training data and the flowchart of the presented model are described as follows.

\subsection{Block Images as Training Data}

As referenced previously, the presented model was trained using a multitude of blockbased images of size $224 \times 224$ pixels in this work. Figure 4 illustrates block images and a lesion contained in a block image. As illustrated in Figure $4 \mathrm{a}, \mathrm{b}$, the white portions represent the same view of a breast, and a mammogram is segmented into overlapping block images from right to left and then top to bottom, with a stride of 36 pixels. Furthermore, a block image where a contained breast occupies no less than $90 \%$ of the block area is chosen as a piece of training data.

As illustrated in Figure 4c, part of a lesion is contained in the block image. Next, a BI-RADS category is assigned to the block image according to the ratio of the areas of the contained lesion to the area of the block, which can be categorized as follows. In Case 1, a block image does not contain a lesion and is assigned as BI-RADS category 1 accordingly. Otherwise, two quantities, ratio $_{\mathrm{B}}$ and $\mathrm{ratio}_{\mathrm{L}}$ are, respectively defined in Case 2 as

$$
\begin{aligned}
& \text { ratio }_{\mathrm{B}}=\frac{\text { Area }_{\mathrm{B}} \cap \text { Area }_{\mathrm{L}}}{\operatorname{Area}_{\mathrm{B}}} \\
& \text { ratio }_{\mathrm{L}}=\frac{\text { Area }_{\mathrm{B}} \cap \text { Area }_{\mathrm{L}}}{\text { Area }_{\mathrm{L}}}
\end{aligned}
$$


where $A r e a_{\mathrm{B}}$ and $A r e a_{\mathrm{L}}$ represent the areas of the block image and the lesion, respectively. Subsequently, if the condition

$$
\text { Condition: }\left(\text { ratio }_{\mathrm{B}} \geq t h r_{\mathrm{B}}\right) \text { or }\left(\text { ratio }_{\mathrm{L}} \geq t h r_{\mathrm{L}}\right)
$$

where $t h r_{\mathrm{B}}=t h r_{\mathrm{L}}=0.5$ are two user-specified thresholds, is true, the block image is then classified as the category of the contained lesion. In Case 3, where there are multiple findings in a block image, check whether the condition in Expression (3) is satisfied. If satisfied, the block image is assigned the highest category in the following hierarchy, from highest to lowest: 5, 4C, 4B, 4A, 0, 3, 2. Otherwise, the block image is assigned as BI-RADS category 1 . All the block images were divided into two parts, as the training and test data, respectively, and Table 3 gives the numbers of these data for each BI-RADS category.

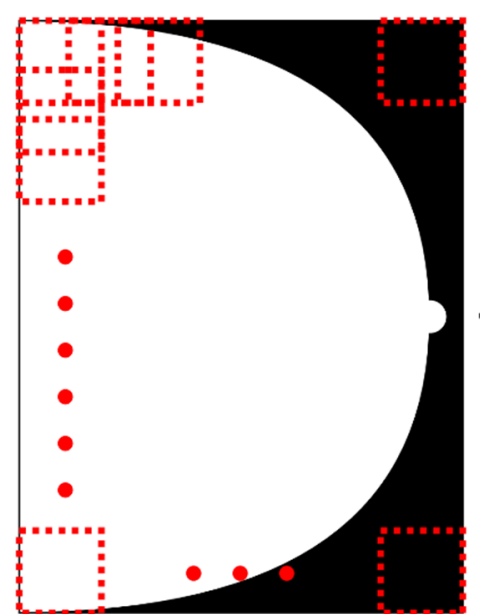

(a)

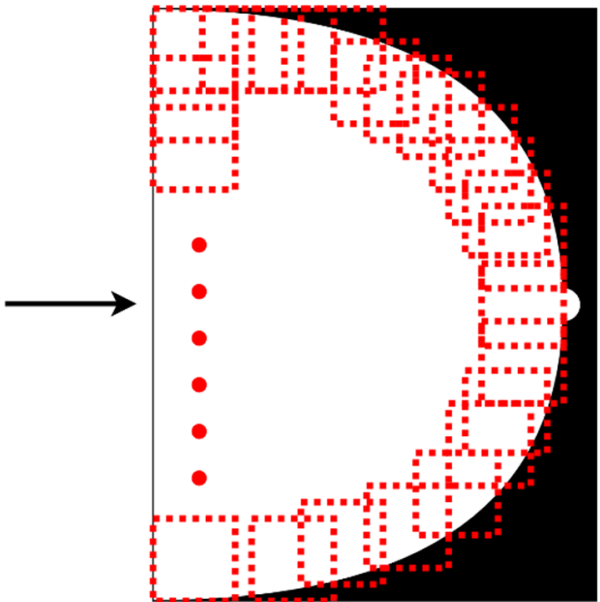

(b)

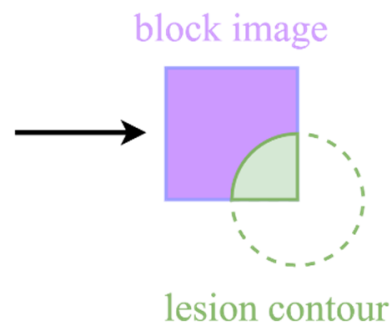

(c)

Figure 4. (a) Overlapping block images, (b) those of (a) selected as training data, and (c) a BI-RADS category assigned to each block image in (b).

Table 3. Numbers of training and test data.

\begin{tabular}{ccc}
\hline BI-RADS & Number of Training Data & Number of Test Data \\
\hline 0 & 42,565 & 10,641 \\
1 & 51,964 & 14,847 \\
2 & 48,294 & 13,322 \\
3 & 47,470 & 12,566 \\
$4 \mathrm{~A}$ & 25,475 & 6369 \\
$4 \mathrm{~B}$ & 28,993 & 7248 \\
$4 \mathrm{C}$ & 36,021 & 9005 \\
5 & 46,741 & 11,685 \\
Sum & 327,523 & 85,683 \\
\hline
\end{tabular}

\subsection{Model Architecture}

The model was built based on one of the state-of-the-art models, EfficientNet [24]. As illustrated in Figure 5, the model, made up of a stem, a body, a head and an output mode, takes a mammogram of size $224 \times 224$ pixels as an input, that is, an input image shape of $224 \times 224 \times 1$. In the Stem module, the input image is firstly normalized to lie between 0 and 1 , and then feature maps are extracted using a $3 \times 3$ convolution layer. Subsequently, high-level feature maps are extracted in the Body module, consisting of 16 mobile inverted bottleneck convolution (MBConv) blocks [25]. Finally, the feature maps are classified in the Head and Output modules. 


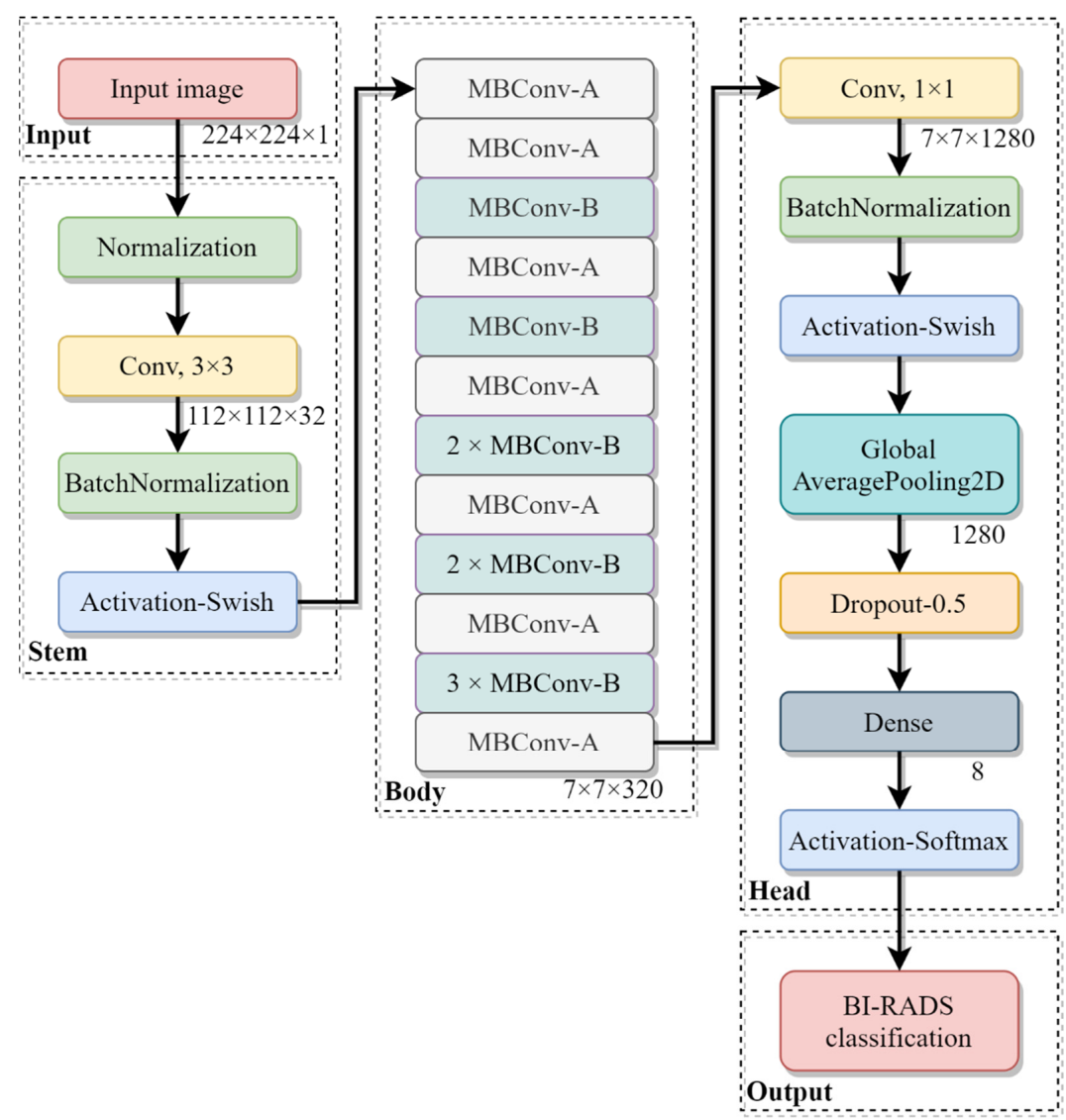

Figure 5. Flowchart of the presented BI-RADS classification model.

A Swish activation function [26], expressed as

$$
\operatorname{Swish}(x, \beta)=x \cdot \operatorname{sigmoid}(\beta x)=\frac{x}{1+e^{-\beta x}}
$$

is used in the Activation-Swish block. As compared with ReLU, the performance of a neural network can be improved in most cases using a Swish activation function. Table 4 summarizes all the modules contained in Figure 5.

Table 4. Summary of each module in the presented model.

\begin{tabular}{cccccc}
\hline Module & Kernel Size & Stride & Expansion Ratio & Parameters & Output Shape \\
\hline Stem & $3 \times 3$ & 2 & - & 416 & (None, 112, 112, 32) \\
MBConv-A & $3 \times 3$ & 1 & 1 & 1544 & $($ None, 112, 112, 16) \\
MBConv-A & $3 \times 3$ & 2 & 6 & 6436 & $($ None, 56, 56, 24) \\
MBConv-B & $3 \times 3$ & 1 & 6 & 11,334 & $($ None, 56, 56, 24) \\
MBConv-A & $5 \times 5$ & 2 & 6 & 16,006 & $($ None, 28, 28, 40) \\
MBConv-B & $5 \times 5$ & 1 & 6 & 32,330 & $($ None, 28, 28, 40) \\
MBConv-A & $3 \times 3$ & 2 & 6 & 38,250 & $($ None, 14, 14, 80) \\
$2 \times$ MBConv-B & $3 \times 3$ & 1 & 6 & 209,960 & (None, 14, 14, 80) \\
MBConv-A & $5 \times 5$ & 1 & 6 & 128,148 & (None, 14, 14, 112) \\
$2 \times$ MBConv-B & $5 \times 5$ & 1 & 622,968 & (None, 14, 14, 112) \\
MBConv-A & $5 \times 5$ & 2 & 6 & 265,564 & (None, $7,7,192)$ \\
$3 \times$ MBConv-B & $5 \times 5$ & 1 & 6 & $1,778,832$ & (None, $7,7,192)$ \\
MBConv-A & $3 \times 3$ & 1 & - & 722,480 & $($ None, $7,7,320)$ \\
Head & $1 \times 1$ & 1 & 424,968 & (None, 8$)$ \\
\hline
\end{tabular}


Figure 6 gives detailed flowcharts of the MBConv-A and B blocks in Figure 5. An MBConv block is mainly composed of an expansion layer, a depthwise layer and a squeeze-andexcitation network (SENet) [27] where $C_{\mathrm{e}}=C_{\mathrm{i}} \times R_{\mathrm{e}}$, and $R_{\mathrm{e}}$ represents the expansion ratio, as tabulated in Table 4. Accordingly, $C_{\mathrm{d}}=C_{\mathrm{i}}$ if $R_{\mathrm{e}}=1$, and $C_{\mathrm{d}}=C_{\mathrm{e}}$ otherwise. Additionally, Table 4 gives the kernel size and the stride for each DepthwiseConv. For stride $=1$, the output shape is equal to the input shape of a feature map, that is, $\left(W_{\mathrm{d}}, H_{\mathrm{d}}\right)=\left(W_{\mathrm{i}}, H_{\mathrm{i}}\right)$. For stride $=2$, the output shape is half of the input shape. The values of the parameters $W_{\mathrm{d}}, H_{\mathrm{d}}$ and $C_{0}$ can be referenced in Table 4 .

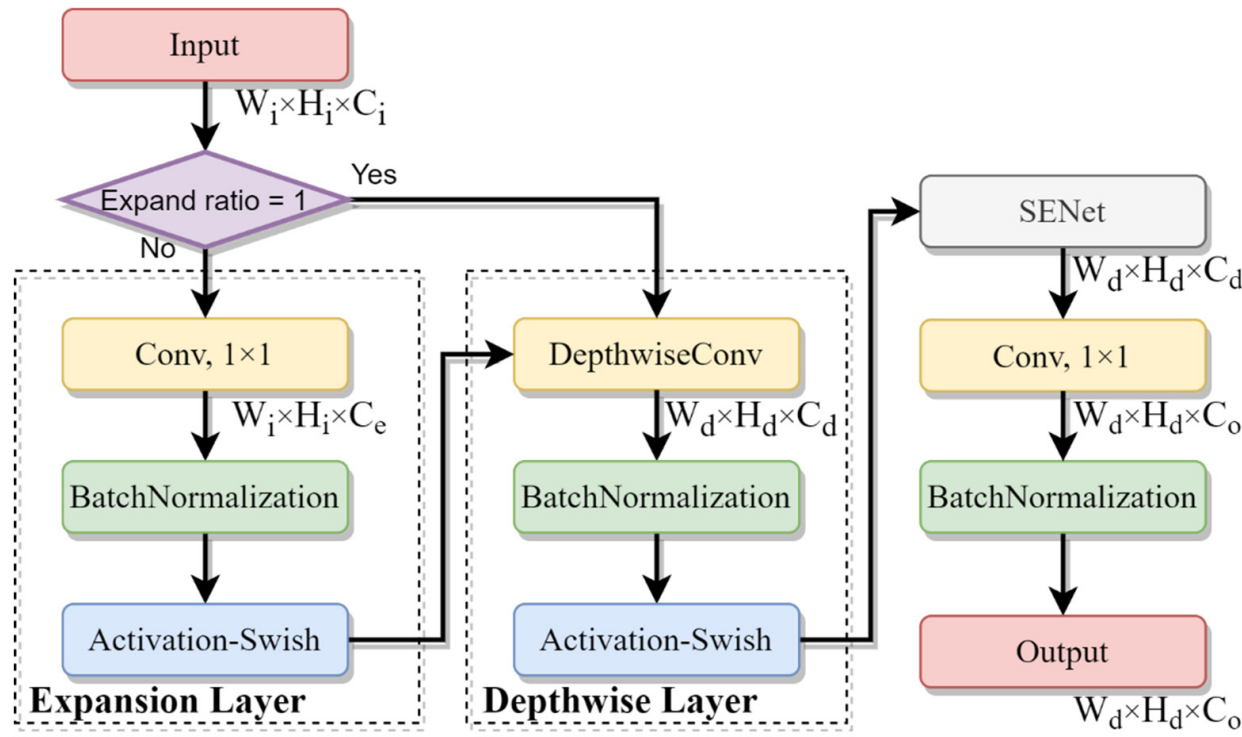

(a)

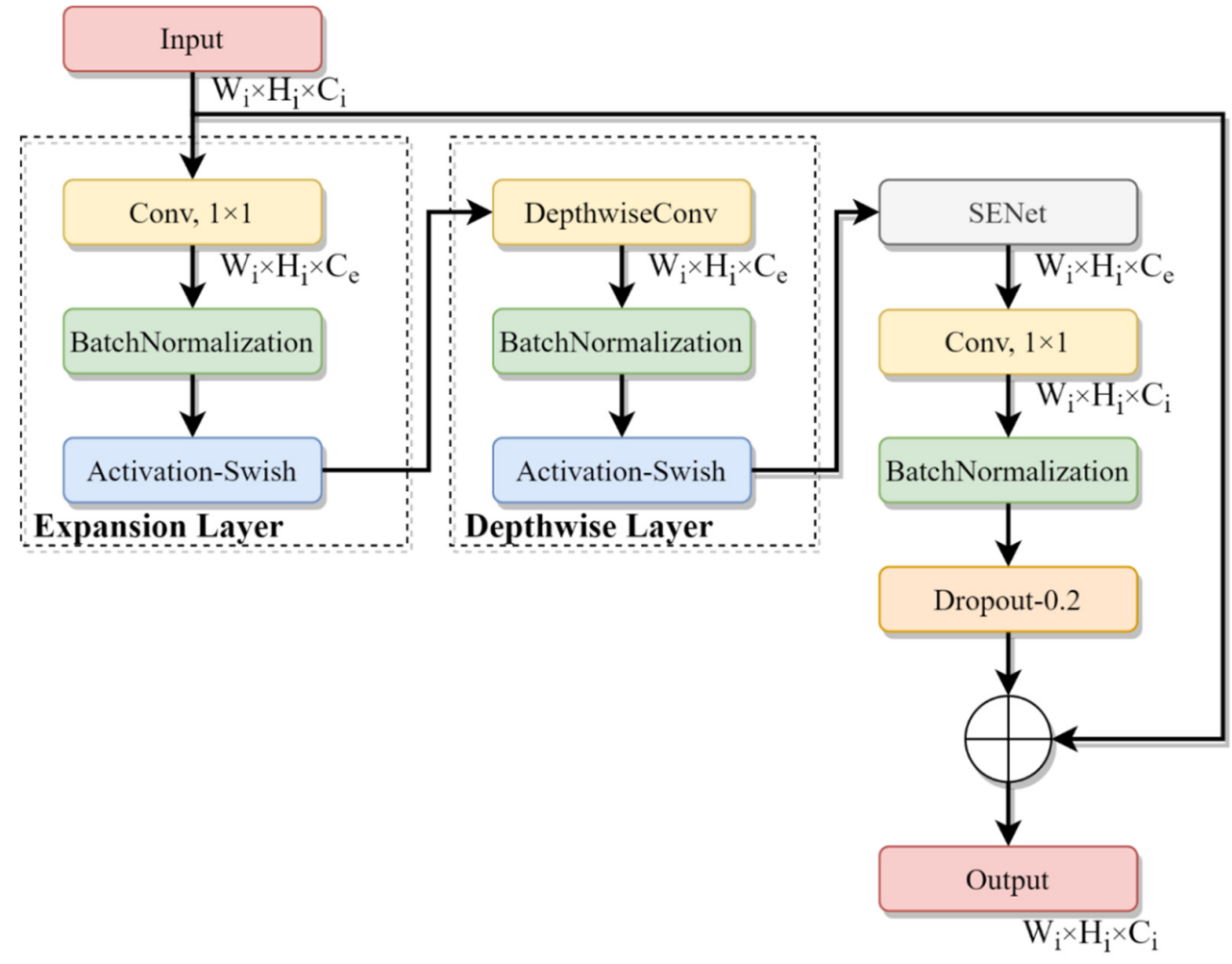

(b)

Figure 6. Flowcharts of (a) the MBConv-A block and (b) the MBConv-B block. 
The SENet module is detailed in Figure 7. A feature map is downsized from $\mathrm{W} \times \mathrm{H} \times \mathrm{C}$ to $1 \times 1 \times \mathrm{C}$ in the squeeze module. To take arbitrary-sized feature map as an input, two fully connected layers are replaced with two convolutional layers with a kernel size of $1 \times 1$ in the excitation module, and $C_{\mathrm{s}}=C_{\mathrm{i}} \times R_{\mathrm{s}}$ where $C_{\mathrm{i}}$ represents the one in the MBConv block, and $R_{\mathrm{S}}$ represents a user-specified ratio that is set to 0.25 . Each channel of the input is weighted non-uniformly by multiplying the input and the output of the excitation module, so as to reflect the significance of each channel feature.

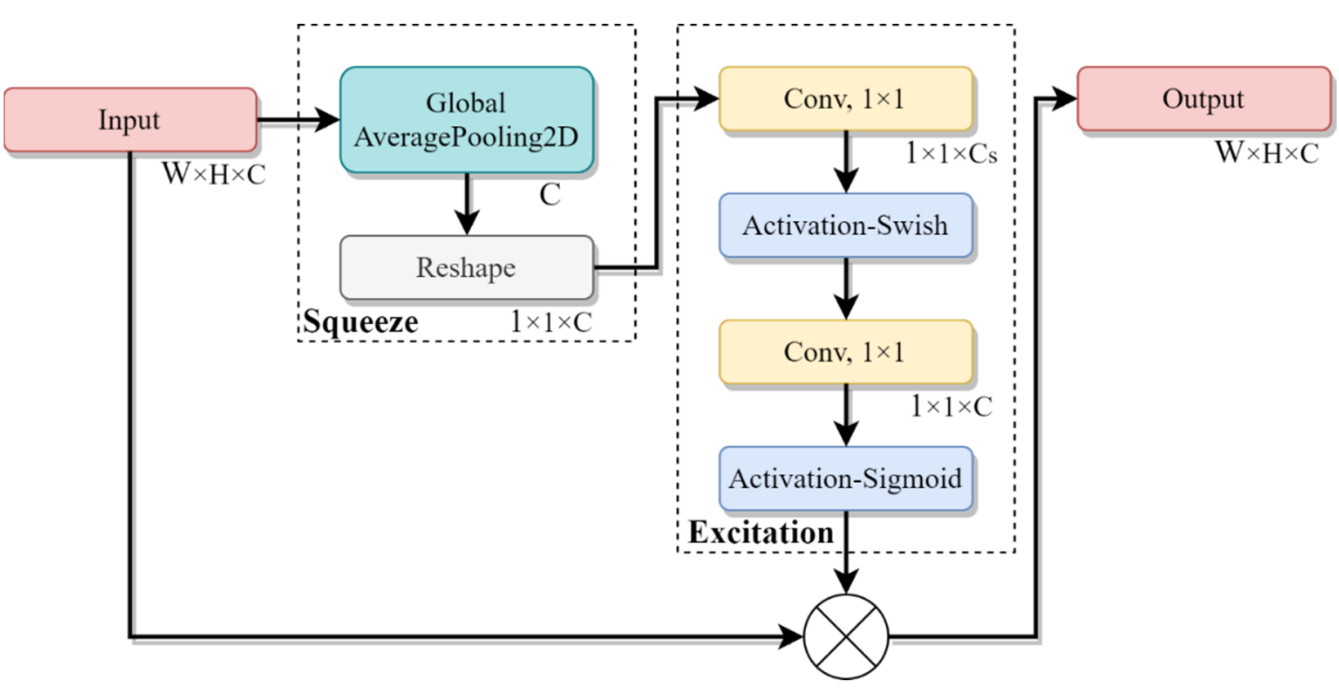

Figure 7. Flowchart of the SENet module.

Finally, a categorical cross-entropy loss function was used to train the model with a batch size of 128 and an epoch of 350, and a Ranger optimizer [28] was also used to improve the training performance. Table 5 lists the development environment of this work.

Table 5. Development environment.

\begin{tabular}{ll}
\hline Programing Language & Python \\
\hline Library & TensorFlow, Keras, numpy, OpenCV, etc. \\
\hline \multirow{2}{*}{ Hardware } & PC (Windows 10 64-bit, Intel i7-10700 2.9 GHz CPU, \\
& 128 GB RAM), graphics card (GeForce RTX 3090) \\
\hline
\end{tabular}

\section{Experimental Results}

A confusion matrix for an eight-class classification system and four performance metrics for each class, including the sensitivity, specificity, precision and F1-score, were evaluated to quantify the model performance. Then, the mean value of each performance metric and the overall accuracy were found.

In Figure 8 , an $8 \times 8$ confusion matrix is used to illustrate how all the performance metrics were evaluated in the case of type 6 (BI-RADS category 4B). True positive (TP) and false positive (FP) are used to represent a lesion that is accurately classified or misclassified as category $4 \mathrm{~B}$, respectively. Likewise, true negative (TN) and false negative (FN) are used to represent a lesion that is accurately classified or misidentified as a category, other than category $4 \mathrm{~B}$, respectively. 


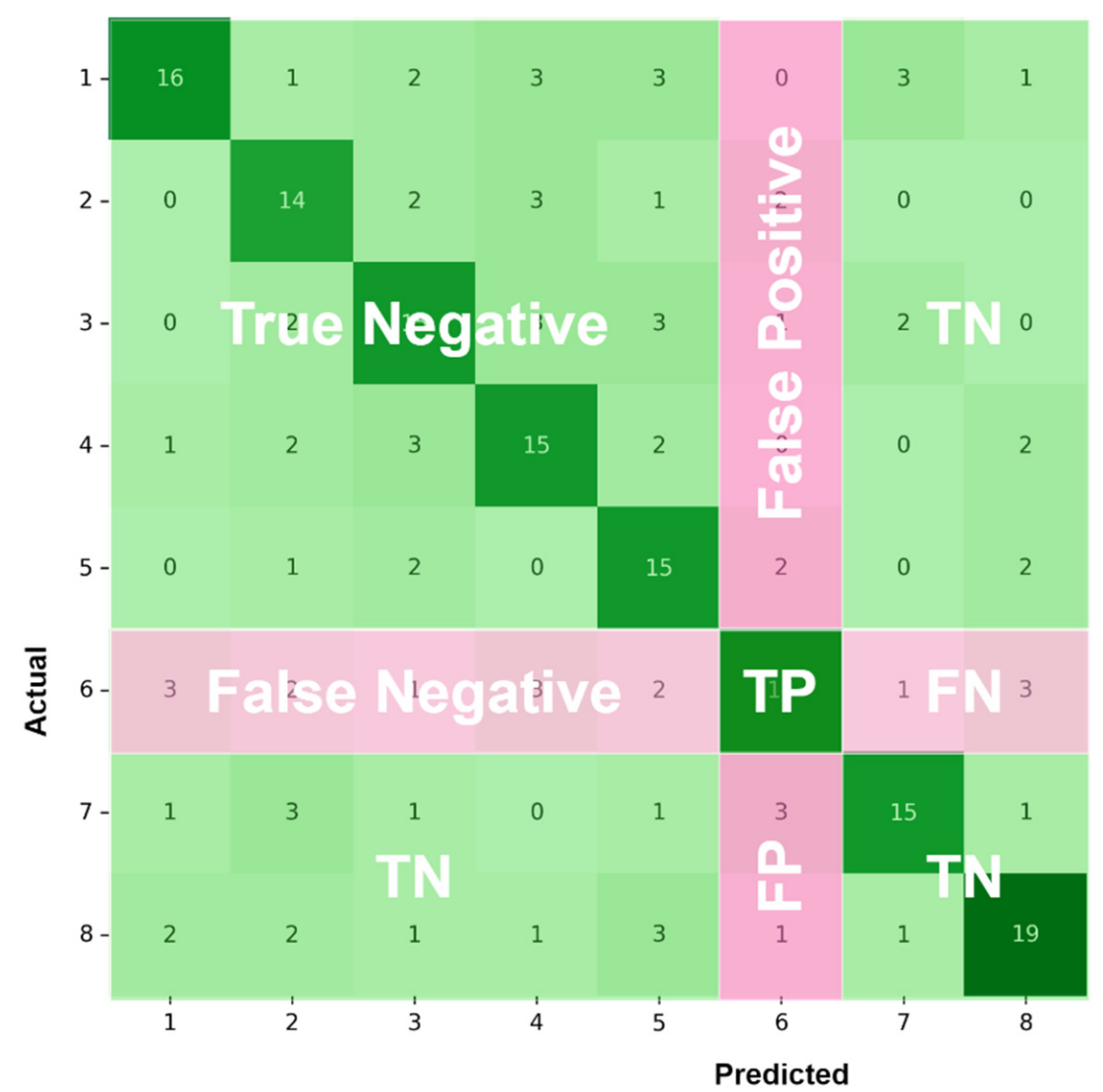

Figure 8. An $8 \times 8$ confusion matrix for illustrative purposes.

Accordingly, all the performance metrics are given, respectively, by

$$
\begin{aligned}
& \text { Sensitivity }_{\mathrm{k}}=T P R_{\mathrm{k}}=T P_{\mathrm{k}} /\left(T P_{\mathrm{k}}+F N_{\mathrm{k}}\right) \\
& \text { Specificity }_{\mathrm{k}}=T N R_{\mathrm{k}}=T N_{\mathrm{k}} /\left(T N_{\mathrm{k}}+F P_{\mathrm{k}}\right) \\
& \text { Precision }_{\mathrm{k}}=P P V_{\mathrm{k}}=T P_{\mathrm{k}} /\left(T P_{\mathrm{k}}+F P_{\mathrm{k}}\right) \\
& \text { F1- } \text { score }_{\mathrm{k}}=2 \times\left(\text { Precision }_{\mathrm{k}} \times \text { Sensitivity }_{\mathrm{k}}\right) /\left(\text { Precision }_{\mathrm{k}}+\text { Sensitivity }_{\mathrm{k}}\right)
\end{aligned}
$$

where $1 \leq k \leq \mathrm{CNum}=8$ and is used to represent that a lesion is classified as category $l=$ the $k$ th element of the hierarchy: $0,1,2,3,4 \mathrm{~A}, 4 \mathrm{~B}, 4 \mathrm{C}, 5$, e.g., category 2 for $k=3$. The sensitivity, specificity and precision are also referred to as the true positive rate (TPR), true negative rate (TNR) and positive predictive value (PPV), respectively. The mean values of the performance metrics in Equations (5)-(8) and the overall accuracy are respectively given by

$$
\begin{gathered}
\text { mean }(x)=\frac{1}{\text { CNum }} \sum_{k=1}^{C N u m} x_{k}, x \in\{\text { Sensitivity, Specificity, Precision, F1-score }\} \\
\text { Accuracy }=\frac{\sum_{k=1}^{C N u m} T P_{k}}{T N u m}=\frac{\sum_{k=1}^{C N u m} T P_{k}}{\sum_{k=1}^{C N u m}\left(T P_{k}+F N_{k}\right)}
\end{gathered}
$$

where TNum represents the number of the test data.

Performance testing was conducted using the 85,683 pieces of test data, as tabulated in Table 3, and led to the confusion matrix in Figure 9 and the performance metrics in Table 6. Subsequently, a receiver operating characteristic (ROC) curve was plotted for each BI-RADS category in Figure 10, and the corresponding area under curve (AUC) value was shown therein. The outperformance of this work was clearly indicated by an average sensitivity 
of $95.31 \%$, an average specificity of $99.15 \%$, an average precision of $94.93 \%$, an average F1-score of $95.11 \%$, an average AUC of $97.23 \%$ and an overall accuracy of up to $94.22 \%$.

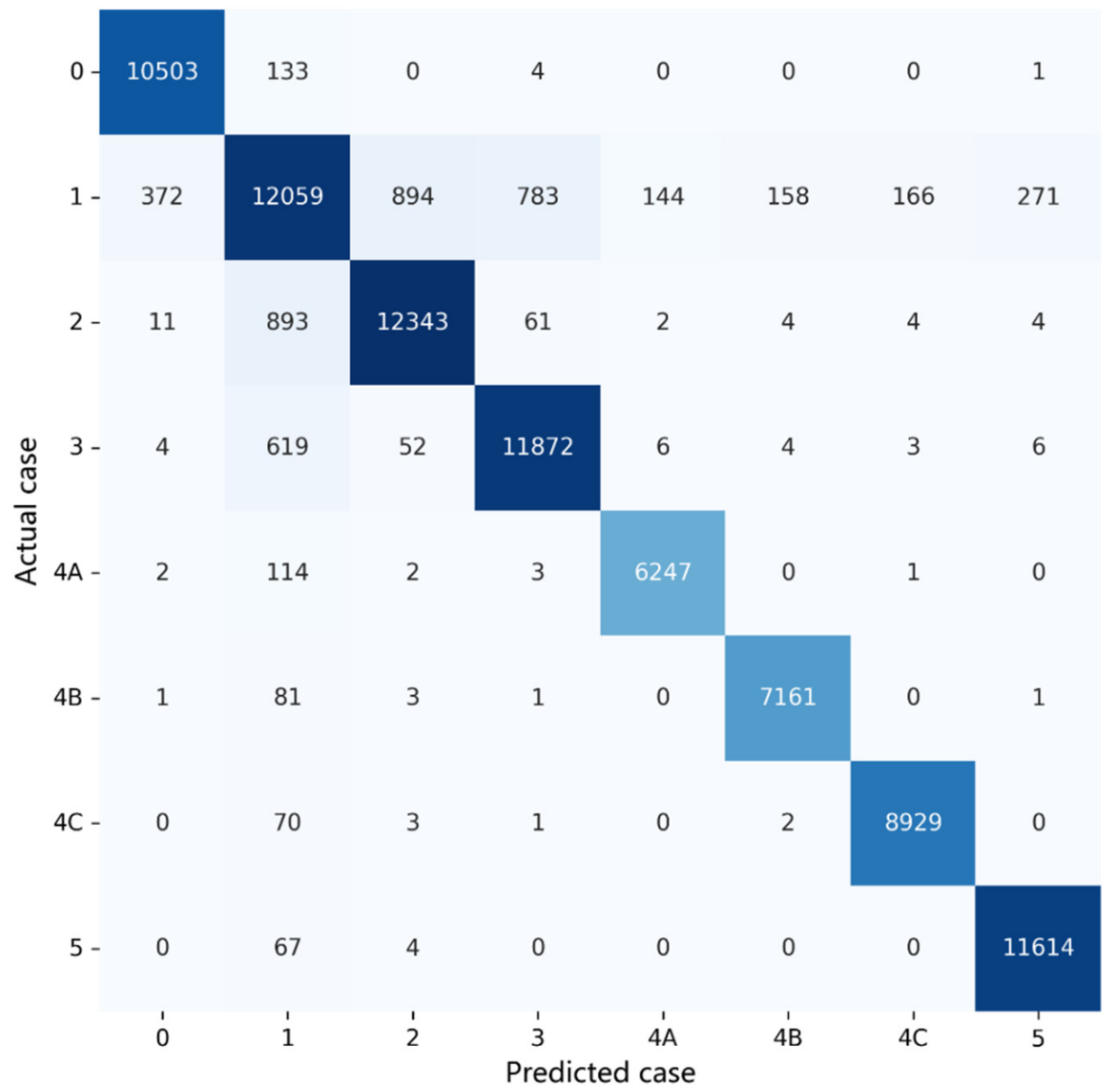

Figure 9. A confusion matrix for performance analysis.

Table 6. Performance metrics of the proposed model.

\begin{tabular}{ccccc}
\hline BI-RADS & Sensitivity (\%) & Specificity (\%) & Precision (\%) & F1-Score (\%) \\
\hline 0 & 98.7031 & 99.4803 & 96.4197 & 97.5481 \\
1 & 81.2218 & 97.2090 & 85.9148 & 83.5024 \\
2 & 92.6513 & 98.6761 & 92.7975 & 92.7243 \\
3 & 94.4772 & 98.8334 & 93.2967 & 93.8832 \\
$4 \mathrm{~A}$ & 98.0845 & 99.8084 & 97.6246 & 97.8540 \\
$4 \mathrm{~B}$ & 98.7997 & 99.7858 & 97.7077 & 98.2507 \\
$4 \mathrm{C}$ & 99.1560 & 99.7731 & 98.0885 & 98.6194 \\
5 & 99.3924 & 99.6176 & 97.6212 & 98.4989 \\
Mean & 95.3107 & 99.1480 & 94.9339 & 95.1101 \\
\hline Accuracy (\%) & \multicolumn{3}{c}{94.2171} \\
\hline
\end{tabular}

In each case of BI-RADS category 0,4A, 4B, 4C and 5 lesions, the sensitivity, specificity and precision exceeded $98 \%, 99 \%$ and $96 \%$, respectively. This validates that such lesions can be well classified using this work, and early-stage breast cancer can be diagnosed more accurately.

In the cases of BI-RADS category 2 and 3 lesions, all the performance metrics lay above $92 \%$, which was slightly below those in the above-referred five cases. The worst performance occurred in the case of BI-RADS category 1 lesions, and the sensitivity and precision hit $81.22 \%$ and $85.91 \%$, respectively, for the following reason. All the lesion-free 
block images were classified as BI-RADS category 1, leading to non-distinctive features that were difficult to diagnose.

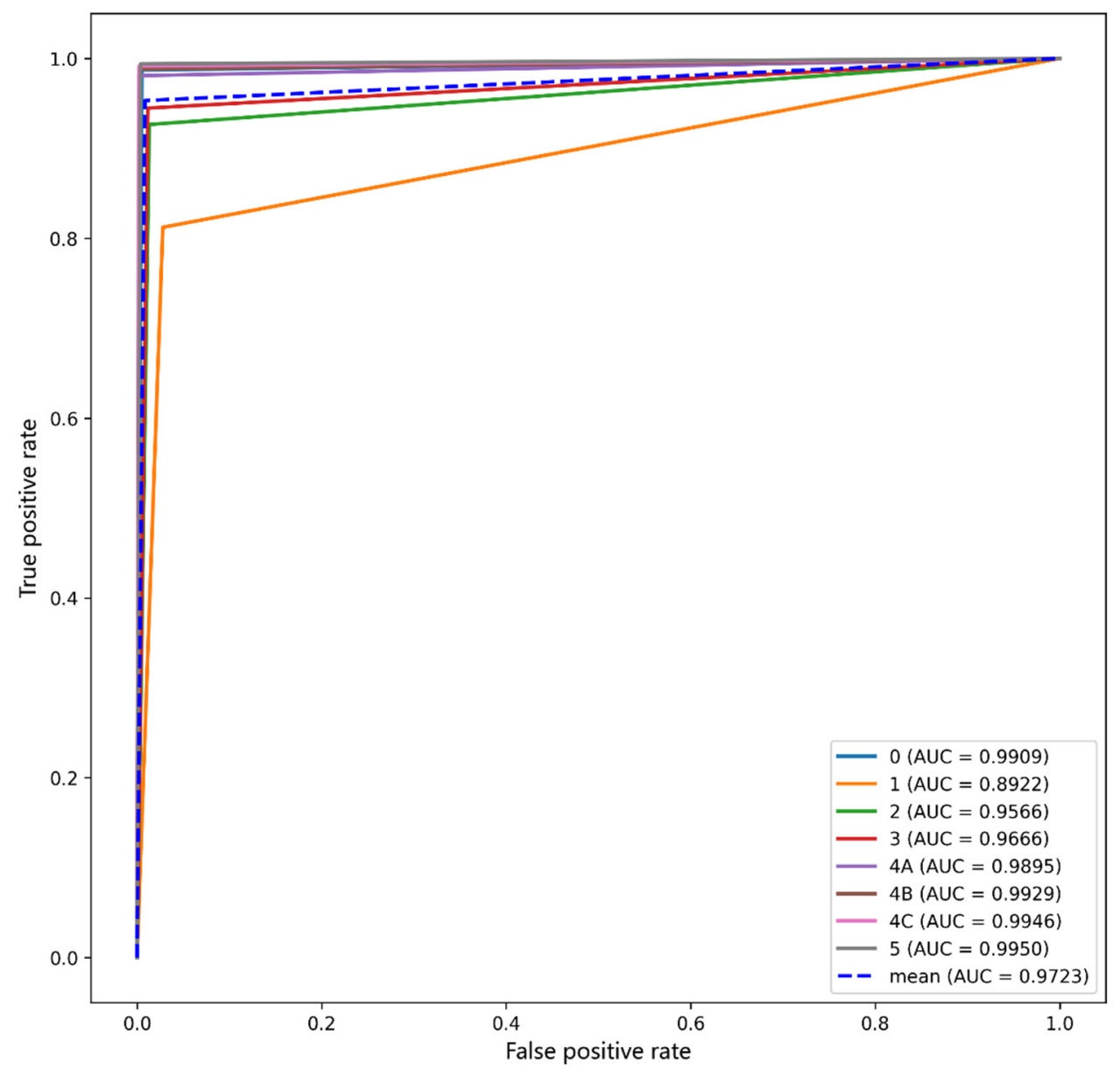

Figure 10. ROC curves of the performance metrics.

A deeper investigation revealed that the sensitivity in the BI-RADS category 1 case was actually a function of the thresholds $t h r_{\mathrm{B}}$ and $t h r_{\mathrm{L}}$ in Equation (3). This is because a block image, classified as BI-RADS category 1, in fact contained a small portion of a lesion in some cases, leading to a negative effect on the training of the presented model. Additionally, each performance metric is also a function of $t h r_{\mathrm{B}}$ and $t h r_{\mathrm{L}}$.

The outperformance of this model was indicated by an overall accuracy of $94.22 \%$, an average sensitivity of $95.31 \%$ and an average specificity of $99.15 \%$. As can be found in Figure 11, there is a good agreement between the red framed ground truth and the blocks, highlighted in color, in each of the mammograms in Figure $11 \mathrm{a}-\mathrm{f}$, where findings were classified as BI-RADS categories 2-5, respectively.

Finally, Table 7 lists the task and performance comparisons between the presented study and previous studies on breast cancer detection in order to reveal the contribution of this work. The Ave_Sen, Ave_Spe and Acc represent the average sensitivity, average specificity and accuracy, respectively. 


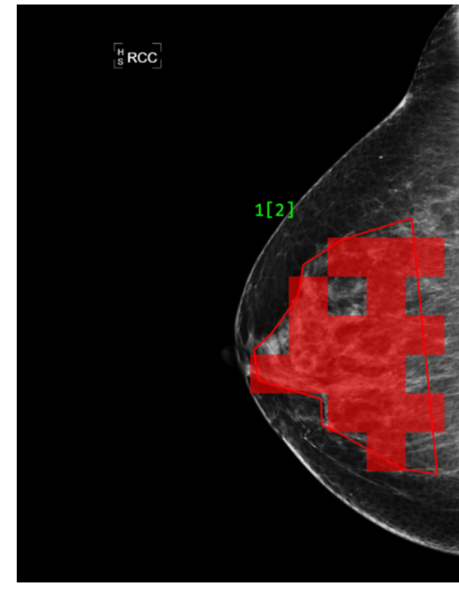

(a)

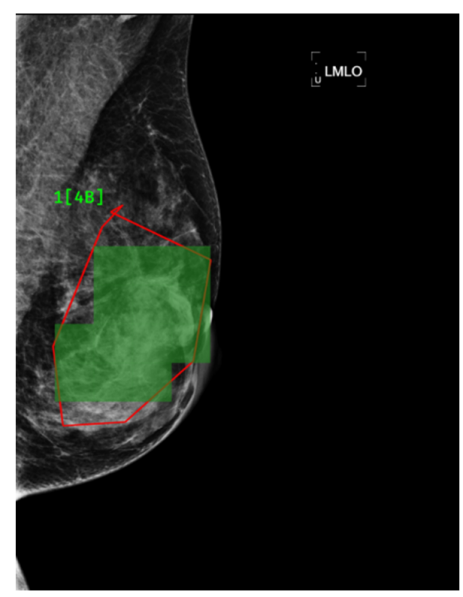

(d)

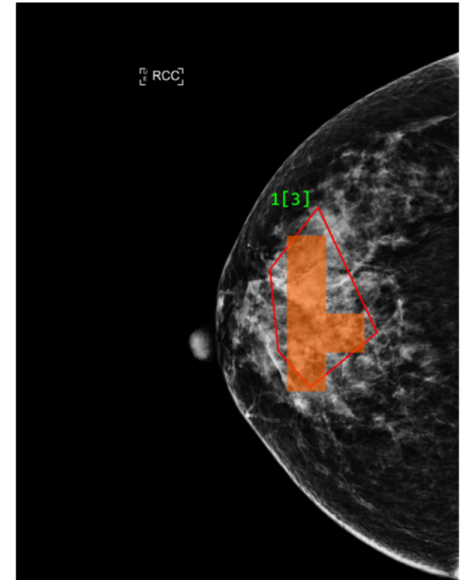

(b)

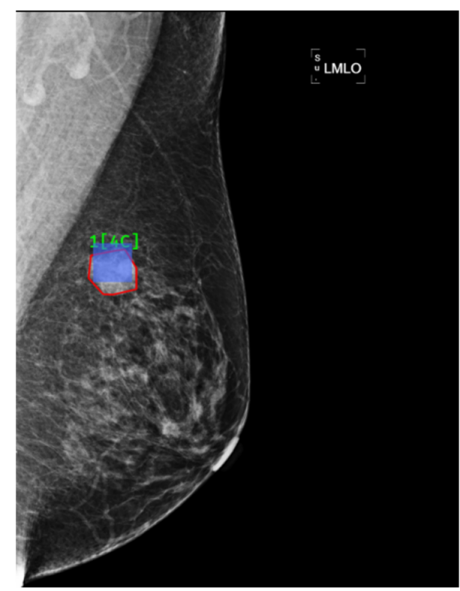

(e)

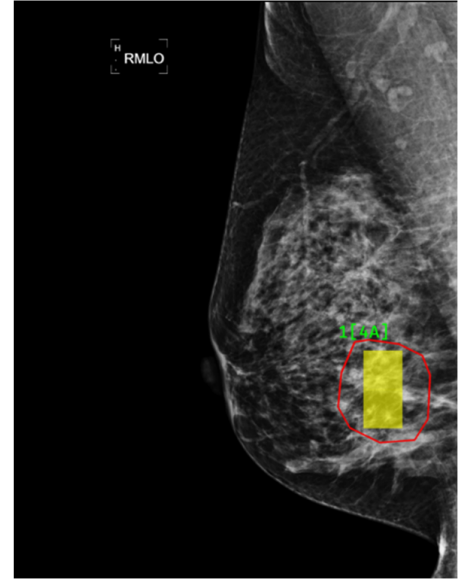

(c)

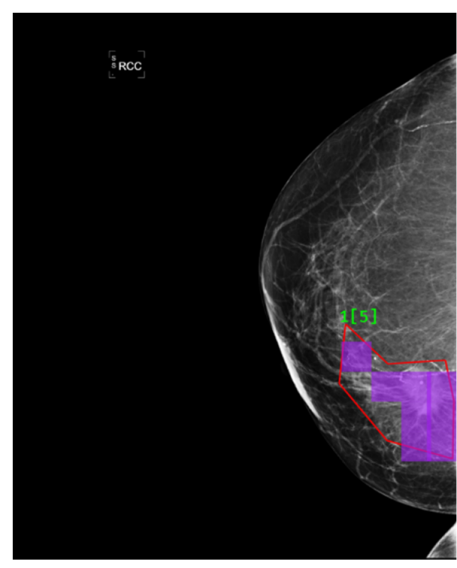

(f)

Figure 11. Comparisons between findings labeled by radiologists (framed in red) and highlighted in color in the cases of BI-RADS category 2, 3, 4A, 4B, 4C and 5 lesions in (a-f), respectively.

Table 7. Task and performance comparisons between the presented study and previous studies on breast cancer detection.

\begin{tabular}{|c|c|c|c|c|c|c|}
\hline $\begin{array}{l}\text { Reference } \\
\text { (Year) }\end{array}$ & Task & Dataset Used & $\begin{array}{l}\text { Ave_Sen } \\
(\%)\end{array}$ & $\begin{array}{l}\text { Ave_Spe } \\
(\%)\end{array}$ & $\begin{array}{l}\text { Acc } \\
(\%)\end{array}$ & AUC \\
\hline $\begin{array}{l}\text { This study } \\
\text { (2022) }\end{array}$ & $\begin{array}{c}\text { Classification of BI-RADS } 0,1,2,3,4 \mathrm{~A}, 4 \mathrm{~B}, \\
4 \mathrm{C}, 5 \\
\text { (8 categories) }\end{array}$ & Private (1490 cases, 5733 images) & 95.31 & 99.15 & 94.22 & 0.972 \\
\hline $\begin{array}{c}{[8]} \\
(2021)\end{array}$ & $\begin{array}{l}\text { Malignancy prediction of BI-RADS } 4 \\
\text { micro-calcifications } \\
\text { ( } 2 \text { classes })\end{array}$ & Private (384 cases, 824 images) & 85.3 & 91.9 & - & 0.910 \\
\hline \multirow{2}{*}{$\begin{array}{l}{[11]} \\
(2021)\end{array}$} & \multirow{2}{*}{$\begin{array}{l}\text { Mass malignancy classification } \\
\text { ( } 2 \text { classes })\end{array}$} & DDSM (2578 cases, 10,312 images) & $\begin{array}{l}89.8 @ 2 \\
\text { FPPI }^{1}\end{array}$ & - & - & - \\
\hline & & Private ( 2807 cases, 11,228 images) & $\begin{array}{l}96.2 @ 2 \\
\text { FPPI }\end{array}$ & - & - & - \\
\hline $\begin{array}{c}{[13]} \\
(2020)\end{array}$ & $\begin{array}{c}\text { BI-RADS 2-5 classification for breast } \\
\text { masses } \\
\text { (4 categories })\end{array}$ & $\begin{array}{c}\text { DDSM } \\
\text { (500 images) }\end{array}$ & 84.5 & 94.25 & 84.5 & - \\
\hline $\begin{array}{c}{[22]} \\
(2019)\end{array}$ & $\begin{array}{c}\text { Normal, benign calcification, } \\
\text { benign mass, malignant calcification, } \\
\text { malignant mass } \\
\text { ( } 5 \text { classes })\end{array}$ & $\begin{array}{c}\text { DDSM + } \\
\text { CBIS-DDSM } \\
\text { (2339 images) }\end{array}$ & - & - & 91 & 0.98 \\
\hline
\end{tabular}

${ }^{1}$ FPPI: false positive per image. 


\section{Conclusions}

This paper presented a DNN-based model to efficiently and reliably locate and classify breast lesions from mammograms. Block-based images, segmented from collected mammograms, were used to adequately train the model, by which the workload of radiologists can be significantly eased, particularly when interpreting mammograms in a large-scale breast cancer screening program. For the first time in the literature, breast lesions can be completely classified into BI-RADS categories $0,1,2,3,4 \mathrm{~A}, 4 \mathrm{~B}, 4 \mathrm{C}$ and 5 . The outperformance of this model was indicated by an overall accuracy of $94.22 \%$, an average sensitivity of $95.31 \%$, an average specificity of $99.15 \%$ and an average AUC of 0.9723 . When applied to breast cancer screening for Asian women, who are more likely to have dense breasts, this model is expected to give a higher accuracy than others in the literature, since it was trained using mammograms taken from Taiwanese women.

It is worth mentioning that this work can provide three benefits for healthcare industries. First, the developed tool can help radiologists with mammographic interpretation in clinical works and can improve the efficiency of mammogram interpretation as well. Second, the workload of radiologists can be reduced remarkably. Third, the tool can assist general physicians with interpreting mammograms due to a shortage of radiologists or breast surgeons in most remote areas.

As the next step, our team aims to upsize the collected dataset so as to better train the model and advance the generalization ability as well. In the meantime, we are making continuous efforts to improve the model performance, particularly in the worst BI-RADS category 1 case. Finally, we will test the generalization ability of this model as an interhospital project.

Author Contributions: Conceptualization, K.-J.T. and S.-H.H.; methodology, C.-Y.Y. and S.-H.H.; software, C.-Y.Y.; validation, M.-C.C., H.-M.L., S.-T.L., J.-H.H. and W.-C.Y.; formal analysis, K.-J.T., C.-Y.Y. and H.-M.L.; investigation, K.-J.T., M.-C.C., W.-C.Y. and C.-Y.Y.; resources, C.-M.H. and S.-H.H.; data curation, M.-C.C., H.-M.L., S.-T.L., J.-H.H. and W.-C.Y.; writing-original draft preparation, C.-Y.Y. and K.-J.T.; writing-review and editing, M.-C.C., H.-M.L. and J.-H.H.; visualization, C.-Y.Y. and S.-T.L.; supervision, C.-M.H., K.-J.T., M.-C.C. and S.-H.H.; project administration, K.-J.T., S.-H.H. and C.-M.H.; funding acquisition, S.-H.H. and C.-M.H. All authors have read and agreed to the published version of the manuscript.

Funding: This research was financially supported by the Ministry of Economic Affairs, Taiwan, under grant number 109-EC-17-A-02-S5-008.

Institutional Review Board Statement: This study was approved by a local institutional review board (EMRP-108-142).

Informed Consent Statement: Informed consent was waived because all the personal identifiable data were deleted.

Data Availability Statement: The data presented in this paper are not publicly available at this time but may be obtained from the corresponding author upon reasonable request.

Conflicts of Interest: The authors declare no conflict of interest.

\section{References}

1. Sung, H.; Ferlay, J.; Siegel, R.L.; Laversanne, M.; Soerjomataram, I.; Jemal, A.; Bray, F. Global Cancer Statistics 2020: GLOBOCAN Estimates of Incidence and Mortality Worldwide for 36 Cancers in 185 Countries. CA-Cancer J. Clin. 2021, 71, 209-249. [CrossRef] [PubMed]

2. Cancer Registry Annual Report, 2018 Taiwan. Health Promotion Administration, Ministry of Health and Welfare, Taiwan, December 2020. Available online: https://www.hpa.gov.tw/EngPages/Detail.aspx?nodeid=1061\&pid=6069 (accessed on 6 October 2021).

3. Chen, Y.P.; Lu, Y.W.; Yang, C.C. Breast cancer trend in Taiwan. MOJ Women's Health 2017, 6, 376-379.

4. Rao, A.A.; Feneis, J.; Lalonde, C.; Ojeda-Fournier, H. A Pictorial Review of Changes in the BI-RADS Fifth Edition. Radiographics 2016, 36, 623-639. [CrossRef] [PubMed] 
5. Aggarwal, R.; Sounderajah, V.; Martin, G.; Ting, D.S.W.; Karthikesalingam, A.; King, D.; Ashrafian, H.; Darzi, A. Diagnostic accuracy of deep learning in medical imaging: A systematic review and meta-analysis. NPJ Digit. Med. 2021, 4, 65. [CrossRef] [PubMed]

6. Abdelrahman, L.; Al Ghamdi, M.; Collado-Mesa, F.; Abdel-Mottaleb, M. Convolutional neural networks for breast cancer detection in mammography: A survey. Comput. Biol. Med. 2021, 131, 104248. [CrossRef] [PubMed]

7. Houssein, E.H.; Emam, M.M.; Ali, A.A.; Suganthan, P.N. Deep and machine learning techniques for medical imaging-based breast cancer: A comprehensive review. Expert Syst. Appl. 2021, 167, 114161. [CrossRef]

8. Liu, H.H.; Chen, Y.H.; Zhang, Y.Z.; Wang, L.J.; Luo, R.; Wu, H.T.; Wu, C.Q.; Zhang, H.L.; Tan, W.X.; Yin, H.K.; et al. A deep learning model integrating mammography and clinical factors facilitates the malignancy prediction of BI-RADS 4 microcalcifications in breast cancer screening. Eur. Radiol. 2021, 31, 5902-5912. [CrossRef]

9. Wang, J.H.; Yang, X.; Cai, H.M.; Tan, W.C.; Jin, C.Z.; Li, L. Discrimination of Breast Cancer with Microcalcifications on Mammography by Deep Learning. Sci. Rep. 2016, 6, 27327. [CrossRef]

10. Stelzer, P.D.; Steding, O.; Raudner, M.W.; Euller, G.; Clauser, P.; Baltzer, P.A.T. Combined texture analysis and machine learning in suspicious calcifications detected by mammography: Potential to avoid unnecessary stereotactical biopsies. Eur. J. Radiol. 2020, 132, 109309. [CrossRef]

11. Yang, Z.; Cao, Z.; Zhang, Y.; Tang, Y.; Lin, X.; Ouyang, R.; Wu, M.; Han, M.; Xiao, J.; Huang, L.; et al. MommiNet-v2: Mammographic multi-view mass identification networks. Med. Image Anal. 2021, 73, 102204. [CrossRef]

12. Cha, K.H.; Petrick, N.; Pezeshk, A.; Graff, C.G.; Sharma, D.; Badal, A.; Sahiner, B. Evaluation of data augmentation via synthetic images for improved breast mass detection on mammograms using deep learning. J. Med. Imaging 2020, 7, 012703. [CrossRef] [PubMed]

13. Boumaraf, S.; Liu, X.B.; Ferkous, C.; Ma, X.H. A New Computer-Aided Diagnosis System with Modified Genetic Feature Selection for BI-RADS Classification of Breast Masses in Mammograms. BioMed Res. Int. 2020, 2020, 7695207. [CrossRef] [PubMed]

14. Akselrod-Ballin, A.; Karlinsky, L.; Alpert, S.; Hashoul, S.; Ben-Ari, R.; Barkan, E. A CNN based method for automatic mass detection and classification in mammograms. Comput. Methods Biomech. Biomed. Eng. Imaging Vis. 2019, 7, 242-249. [CrossRef]

15. Wu, N.; Phang, J.; Park, J.; Shen, Y.Q.; Huang, Z.; Zorin, M.; Jastrzebski, S.; Fevry, T.; Katsnelson, J.; Kim, E.; et al. Deep Neural Networks Improve Radiologists Performance in Breast Cancer Screening. IEEE Trans. Med. Imaging 2020, 39, 1184-1194. [CrossRef] [PubMed]

16. Sasaki, M.; Tozaki, M.; Rodriguez-Ruiz, A.; Yotsumoto, D.; Ichiki, Y.; Terawaki, A.; Oosako, S.; Sagara, Y.; Sagara, Y. Artificial intelligence for breast cancer detection in mammography: Experience of use of the ScreenPoint Medical Transpara system in 310 Japanese women. Breast Cancer 2020, 27, 642-651. [CrossRef]

17. Agnes, S.A.; Anitha, J.; Pandian, S.I.A.; Peter, J.D. Classification of Mammogram Images Using Multiscale all Convolutional Neural Network (MA-CNN). J. Med. Syst. 2020, 44, 30. [CrossRef]

18. Ribli, D.; Horvath, A.; Unger, Z.; Pollner, P.; Csabai, I. Detecting and classifying lesions in mammograms with Deep Learning. Sci. Rep. 2018, 8, 4165. [CrossRef]

19. Cruz, T.N.; Cruz, T.M.; Santos, W.P. Detection and Classification of Lesions in Mammographies Using Neural Networks and Morphological Wavelets. IEEE Lat. Am. Trans. 2018, 16, 926-932. [CrossRef]

20. Carneiro, G.; Nascimento, J.; Bradley, A.P. Automated Analysis of Unregistered Multi-View Mammograms With Deep Learning. IEEE Trans. Med. Imaging 2017, 36, 2355-2365. [CrossRef]

21. Shen, L.; Margolies, L.R.; Rothstein, J.H.; Fluder, E.; McBride, R.; Sieh, W. Deep learning to improve breast cancer detection on screening mammography. Sci. Rep. 2019, 9, 12495. [CrossRef]

22. Tang, C.M.; Cui, X.M.; Yu, X.; Yang, F. Five Classifications of Mammography Images Based on Deep Cooperation Convolutional Neural Network. Am. Sci. Res. J. Eng. Technol. Sci. 2019, 57, 10-21.

23. American College of Radiology (ACR). ACR BI-RADS Atlas, 5th ed.; ACR: Reston, VA, USA, 2013

24. Tan, M.; Le, Q.V. EfficientNet: Rethinking Model Scaling for Convolutional Neural Networks. arXiv 2020, arXiv:1905.11946.

25. Sandler, M.; Howard, A.; Zhu, M.; Zhmoginov, A.; Chen, L.C. MobileNetV2: Inverted Residuals and Linear Bottlenecks. arXiv 2019, arXiv:1801.04381.

26. Ramachandran, P.; Zoph, B.; Le, Q.V. Searching for Activation Functions. arXiv 2017, arXiv:1710.05941.

27. Hu, J.; Shen, L.; Albanie, S.; Sun, G.; Wu, E. Squeeze-and-Excitation Networks. IEEE Trans. Pattern Anal. Mach. Intell. 2020, 42, 2011-2023. [CrossRef]

28. Wright, L.; Demeure, N. Ranger21: A synergistic deep learning optimizer. arXiv 2021, arXiv:2106.13731. 